\title{
Deciphering the star codings: astrocyte manipulation alters mouse behavior
}

\author{
Keebum Park ${ }^{1}$ and Sung Joong Lee ${ }^{2}$
}

\begin{abstract}
Astrocytes occupy a vast area within the central nervous system (CNS). Despite their abundance, the functional role of astrocytes in vivo has only begun to be uncovered. Astrocytes were typically thought to be involved in pathophysiological states. However, recent studies have shown that astrocytes are actively involved in cell signaling in normal physiological states; manipulating various aspects of astrocytic cell signaling in vivo has revealed that astrocytes are key players in controlling healthy behavior in the absence of pathophysiology. Unfortunately, the study of astrocyte function is often limited by the number of approaches available due to our lack of understanding of cell physiology. This review summarizes recent studies in which altered astrocyte signaling capacity resulted in dramatic changes in behavior. We not only discuss the methodologies available to manipulate astrocytes but also provide insights into the behavioral roles of astrocytes in the CNS.
\end{abstract}

\section{Introduction}

Astrocytes, or "star-shaped" cells, which are as abundant in our CNS as neurons, densely and homogeneously populate the brain, spinal cord, and retina ${ }^{1,2}$. Although astrocytes are abundantly distributed across the entire brain, they have traditionally been regarded as "support cells" that play metabolic and homeostatic roles for nearby neurons ${ }^{3}$. The first evidence that neurons and astrocytes exchange functional signaling was presented $\sim 30$ years ago ${ }^{4-6}$. Later studies found that the star-shaped cells are particularly active in processing pain and inflammation ${ }^{7-9}$. Since then, studies have mainly focused on the role of astrocytes within the context of the brain's pathophysiological states ${ }^{10,11}$. However, their role in physiological brain function in vivo has not been investigated for a while.

Over the last decade, there has been accumulating evidence that astrocytes actively participate in the brain's

\footnotetext{
Correspondence: Keebum Park (kbpark91@snu.ac.kr) or

Sung Joong Lee (sjlee87@snu.ac.kr)

${ }^{1}$ Interdisciplinary Program in Neuroscience, College of Natural Sciences,

Seoul National University, Seoul, Republic of Korea

2Department of Physiology and Neuroscience, Dental Research Institute,

Seoul National University School of Dentistry, Seoul, Republic of Korea
}

physiological activities. Recent studies have also recognized the importance of the functional and regulatory roles that astrocytes play under normal physiological conditions. The final outputs that produce one's behavior are generally accepted to be neuronal; however, astrocytes often critically modulate this final output. This relationship requires dynamic cell signaling between neurons and astrocytes. Morphologically, astrocytes have numerous processes that are in close proximity to neurons, other astrocytes, and blood vessels ${ }^{12}$. Astrocytic processes make contact with neuronal synapses and form tripartite synapses. A single astrocyte can make as many as 140,000 contacts with neuronal synapses ${ }^{13}$. Astrocytes actively exchange signals with nearby cells to complement and modulate neuronal communications in their vicinity. They often alter the electrophysiological properties of the surrounding area $^{14}$. Therefore, it would not be unexpected for some of the astrocyte's modulatory effects to influence behavioral outcomes in vivo. The hypothesis that astrocytes are directly involved in brain activity is further supported by recent studies showing how these cells are transcriptionally, translationally, morphologically, and functionally diverse across brain regions ${ }^{15}$. This evidence is important for understanding the more active 
roles of astrocytes under various physiological states. Efforts to uncover the effects of astrocytes have yielded valuable insights and tools for researchers. Consequently, astrocytes are no longer regarded as passive auxiliary support cells that function uniformly throughout the CNS.

Despite such advances and endeavors, however, a large portion of astrocyte physiology remains vague, and it is unclear how astrocytes process information to affect behavior ${ }^{16}$. Therefore, studies are needed to characterize astrocyte functions as well as their roles in the modulation of various behaviors. In this review, we highlight recent studies in which artificial disruption or enhancement of astrocyte cell signaling capacity in vivo resulted in immediate behavioral changes. These studies used experimental designs including but not limited to pharmacological, genetic, optogenetic, and chemogenetic approaches to specifically target astrocyte signaling processes. Additionally, we summarize the evidence for and provide current perspectives on the roles that astrocytes play in cell signaling and their critical impacts on healthy behaviors in rodents.

\section{Astrocytic cell signaling}

Manipulating astrocytes requires a profound understanding of their signaling processes because each step may be important in understanding astrocyte function. Astrocytes are not electrically excitable cells; nevertheless, they express wide range of functional neurotransmitter receptors ${ }^{17}$. One of the most prominent responses that astrocytes have to an external input is elevating intracellular $\mathrm{Ca}^{2+}$ levels. Following the first report of astrocytic $\mathrm{Ca}^{2+}$ elevation ${ }^{18}$, there have been multiple attempts to understand the dynamics, origins, and implications of this process. These attempts have included the use of fluorescent $\mathrm{Ca}^{2+}$ indicator dyes, such as fura-2, rhod-2, or fluo4 , and genetically encoded calcium indicators (GECIs), such as the calmodulin-containing GCaMP series. Fluorescent $\mathrm{Ca}^{2+}$ indicators have been used to uncover several important physiological properties of astrocytes ${ }^{19,20}$ and are still the primarily used indicators in in vitro studies. However, some studies have reported difficulties in maintaining uniform levels of the dyes across the entire cell over an extended period of time ${ }^{21}$. These dyes have also been reported to be toxic to cells ${ }^{22}$. Given the clear limitations to this approach, most in vivo studies use GECIs to measure $\mathrm{Ca}^{2+}$ elevations in astrocytes. GECIs are stably expressed over time and do not alter normal signaling levels or have detrimental effects on cells; therefore, they are particularly suitable for long-term in vivo recordings ${ }^{23}$. As the use of GCaMPs has become the standard calcium-measuring method for both neurons and astrocytes, the series has been continually adjusted to improve the resolution, localization, and stability ${ }^{24,25}$.
While traditional GECIs allowed researchers to only observe a single $\mathrm{Ca}^{2+}$ dynamics, recent technological advancements have enabled multilayered approaches to imaging and measuring $\mathrm{Ca}^{2+}$ dynamics. Modified GECIs $^{24}$ keep similar wavelengths from interfering with one another and are less sensitive to artificial stimuli such as light. Researchers are also able to mark neurons and astrocytes simultaneously using multicolored $\mathrm{GECIs}^{25}$ to deduce temporal associations between regional cell types.

Astrocytic $\mathrm{Ca}^{2+}$ arises from multiple sources. A major source of $\mathrm{Ca}^{2+}$ elevation is the endoplasmic reticulum, in which $\mathrm{Ca}^{2+}$ release is triggered via activated inositol triphosphate $\left(\mathrm{IP}_{3}\right)$ receptors ${ }^{26}$. Interestingly, both $\mathrm{Gq}$ coupled and Gi-coupled G-protein coupled receptors (GPCRs) have been shown to elevate $\mathrm{Ca}^{2+}$ levels in astrocytes $^{27-29}$. While several studies that targeted the GPCR-IP 3 pathway to control astrocytic $\mathrm{Ca}^{2+}$ activity have failed to identify any noticeable physiological effects $^{30,31}$, other studies performed with Ip3r2 ${ }^{-1-}$ mice have found that this receptor is mainly responsible for somatic $\mathrm{Ca}^{2+}$ signals but not for $\mathrm{Ca}^{2+}$ signaling in cell processes $^{32}$. This implies that there are other factors contributing to $\mathrm{Ca}^{2+}$ elevation in astrocytes, particularly in cell processes. Furthermore, the presence of spontaneous and seemingly random patches of $\mathrm{Ca}^{2+}$ in astrocytes is mediated by ion influx through transient receptor potential (TRP) A1 and V1 channels ${ }^{33,34}$. The TRPA1 channel is also responsible for maintaining resting $\mathrm{Ca}^{2+}$ levels ${ }^{33}$. More recently, it was shown that mitochondria mediate localized $\mathrm{Ca}^{2+}$ transients in astrocyte microdomains ${ }^{35}$. Taken together, these findings demonstrate that astrocyte $\mathrm{Ca}^{2+}$ signaling is highly dynamic. Assessing the implications of astrocyte function requires analysis with more spatiotemporal specificity, as well as comparisons of spontaneous and evoked, fast and slow, and global and focal signaling ${ }^{36}$. Nevertheless, studies that address the sources of astrocytic $\mathrm{Ca}^{2+}$ provide important information on potential targets for manipulating the astrocytic $\mathrm{Ca}^{2+}$ response.

After astrocytes receive signals and internally process them (primarily through $\mathrm{Ca}^{2+}$ elevation), they often emit signals to nearby cells. There is strong evidence that astrocytes are able to release signaling substances ${ }^{16}$ and that strong $\mathrm{Ca}^{2+}$ elevation precedes gliotransmitter release $^{36}$. Gliotransmitters are neuroactive molecules released from astrocytes. These include glutamate, Dserine, ATP, and more. Upon their release into extracellular medium, gliotransmitters can exert an influence on local neurotransmission ${ }^{37}$. Findings suggest that astrocytes utilize SNARE-mediated vesicular exocytosis mechanisms for gliotransmission. Electron microscopy studies have detected gliotransmitters in labeled astrocytic vesicles ${ }^{38}$. In addition, soluble NSF attachment protein receptor (SNARE) isoforms have been identified in 
astrocytes, which also supports the vesicular-release model $^{39}$. While the $\mathrm{Ca}^{2+}$ dynamics in astrocytes seem mainly asynchronous and oscillatory, one study demonstrated that gliotransmitter release is much more strongly correlated with a single large calcium elevation than with multiple small oscillatory waves ${ }^{40}$. Astrocytes have been shown to release D-serine to modulate long-term potentiation (LTP) in the CA1 region of the hippocampus ${ }^{41}$. They also release glutamate to induce timing-dependent long-term depression in the somatosensory cortex ${ }^{42}$ and release adenosine triphosphate (ATP) in response to mechanical stretch in the hippocampus ${ }^{43}$. It is not yet known whether a single astrocyte can release multiple neurotransmitters simultaneously or whether these cells can distinguish different input signals by differentiating their output transmitters. It also remains to be determined whether the release mechanism for each gliotransmitter is unique ${ }^{44}$. When astrocytes become reactive after injury or inflammation, their morphology and $\mathrm{Ca}^{2+}$ signaling patterns change drastically. Following a pathophysiological state in the brain, the amplitude and frequency of $\mathrm{Ca}^{2+}$ signals generally tend to increase ${ }^{43}$. In some models, astrocytic signals occur earlier than neuronal signals ${ }^{38}$. Although the detailed mechanism through which reactive astrocytes display heightened signaling is not completely understood, there is evidence that the aforementioned $\mathrm{IP}_{3}$ and TRPA1 pathways contribute in some way ${ }^{43}$. Such signaling complexities provide multiple areas of possible investigation to elucidate the consequences of astrocyte functions.

The approaches that are discussed in this review either enhance or impair one of the signaling pathways described above (Fig. 1). In genetic knockout models, such as Ip3r2 ${ }^{-1-}$ mice and $\mathrm{Trpa}^{-1-}$ mice, some of the known contributors to $\mathrm{Ca}^{2+}$ elevation in astrocytes are eliminated. Other studies genetically have introduced artificial pumps in astrocyte membranes to eliminate all intracellular calcium ions ${ }^{45}$. SNARE activity can also be modulated to regulate gliotransmission. Manipulation of designer receptors exclusively activated by designer drugs (DREADDs) has also been employed. These nonendogenous receptors are coupled with either Gq(hM3Dq) or $\mathrm{Gi}(\mathrm{hM} 4 \mathrm{Di})$ to modulate astrocyte activity. However, there are reports that the $\mathrm{Ca}^{2+}$ levels in astrocytes increase in response to both $\mathrm{Gq}$ and $\mathrm{Gi}^{28,29}$, albeit to different degrees, and the specific differences between the two have not yet been characterized in vivo. Lightsensitive channelrhodopsin family proteins have also been used to study astrocytes. Although astrocytes are not electrically excitable, cation channels greatly increase $\mathrm{Ca}^{2+}$ levels in astrocytes ${ }^{46}$. Recently, the G-proteincoupled photopigment melanopsin was used for precise optogenetic astrocyte activation ${ }^{47}$. Admittedly, some of the methods employed by recent studies, especially optogenetic manipulation, have been brought into question $^{48,49}$. As our understanding of the intercellular signals in astrocytes improves, there will be more layers to explore beyond those able to be studied with the currently available investigative approaches.

\section{Astrocyte manipulation in vivo \\ Astrocytes in circadian rhythm and sleep}

One of the first behaviors that is suspected to be under astrocyte control is circadian rhythm, in part because the earliest understanding of astrocytes suggested that they regulate homeostatic control. The mammalian circadian system is derived from oscillatory regulation of transcription factors. It is imperative for organisms to maintain this timekeeping system to maintain concordance with the daily cycle. The circadian rhythm is thought to be supervised by neurons in the suprachiasmatic nucleus $(\mathrm{SCN})$. These neurons express the transcription factors brain and muscle Arnt-like protein-1 (BMAL1) and circadian locomotor output cycles kaput (CLOCK) in a feedback loop fashion ${ }^{50}$. These neurons have been the focus of many circadian rhythm studies, while astrocytes have largely been overlooked. However, there is clear evidence that astrocytes rhythmically express circadian oscillators such as Bmal1 as well ${ }^{51}$. Astrocytes are also able to receive circadian inputs from nearby $\mathrm{SCN}$ neurons and release gliotransmitters in response ${ }^{51}$. It was uncertain whether astrocyte rhythmic activity led to circadian behavior until the recent work by Brancaccio et al. ${ }^{52}$. They used cryptochrome circadian regulator $1 / 2$ (Cry1/2)-null mice, which are circadian-incompetent. Since CRY1 and CRY2 are necessary for a transcription-translation negative feedback loop (TTFL), Cry1/2-null mice have dysfunctional TTFL cycles and display no circadian behavior $^{52}$. Cry1 was then selectively restored in SCN astrocytes or neurons by injecting Cre-dependent AAVs encoding Cry1::EGFP with GFAP-Cre or Syn-Cre AAV constructs. As expected, complementing Cry1 in SCN neurons quickly restored the TTFL, and the mice began exhibiting $\sim 26-\mathrm{h}$ behavior cycles ${ }^{52}$. Interestingly, complementing Cry1 only in the SCN astrocytes of Cry1/2null mice was sufficient to induce the TTFL and restore circadian behavior ${ }^{52}$. This effect was largely blocked by inhibiting Cx43, an astrocyte-specific connexin hemichannel that is responsible for gliotransmitter release. In addition, treatment with the NMDA receptor antagonist DQP-1105 also blocked the rescue effect. These results suggest that astrocytic glutamate signaling is responsible for the restoration of circadian behavior ${ }^{52}$. In accordance with this, Tso et al. ${ }^{53}$ also focused on the function of SCN astrocytes in circadian signaling by specifically knocking out the circadian oscillator gene Bmal1 in SCN astrocytes using clustered regularly interspaced short palindromic repeats (CRISPR) with CRISPR-associated 


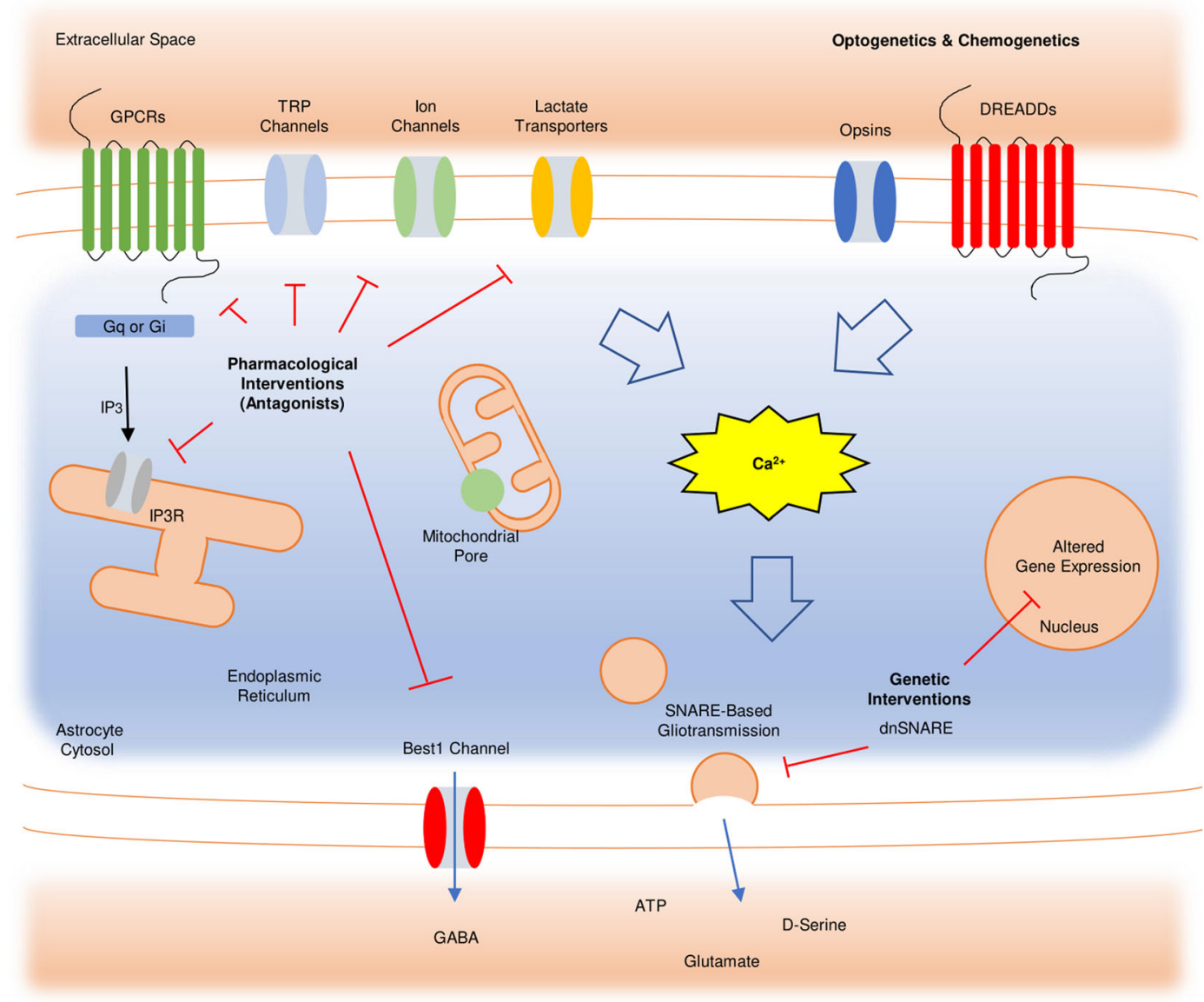

Fig. 1 Astrocyte signaling pathways and intervention points. This schematic outlines some of the major signaling pathways in astrocytes. Changes in astrocytic intracellular $\mathrm{Ca}^{2+}$ levels depend on a number of activities by receptors, channels, pores, and other cellular components. Therefore, each component or process illustrated in this figure is also a possible intervention point to control intracellular $\mathrm{Ca}^{2+}$ levels. The components are roughly classified into several categories. Manipulating the activity of membrane proteins can be done using antagonists and blockers. GPCRs include $m A C h R, G A B A_{B}$, and NMDAR, and the GPCR-IP 3 pathway can also be regulated by controlling $I_{3} R_{2}$ activity. Other membrane proteins, such as TRP channels, ion channels, and voltage-gated calcium channels (not shown), are also responsible for the Ca ${ }^{2+}$ response in astrocytes. Elevated astrocyte $\mathrm{Ca}^{2+}$ often leads to altered gene expression and SNARE-based gliotransmission. Although the gliotransmitter released varies between brain regions, dominant-negative SNARE proteins can be utilized to restrict gliotransmission.

protein 9 (Cas9). Although astrocytes were shown to be functional circadian oscillators under normal conditions, the loss of Bmal1 disrupted the TTFL in astrocytes and significantly lengthened the circadian period ${ }^{53}$. Similarly, the presence of the casein kinase 1 epsilon (CK1ع) tau mutation specifically in astrocytes (Aldh1L1-CK1 $1 \varepsilon^{\mathrm{tau} /+}$ ) shortened the circadian period. Restoring this mutation in the SCN area somewhat rescued the phenotype ${ }^{53}$. This study did not specifically describe how astrocytes control the circadian rhythm; however, Brancaccio et al. predicted that it is partially due to a disruption in astrocyte-neuron signaling in the $\mathrm{SCN}^{52}$. Such an interaction also appears to occur in other species, as the astrocyte-specific expression of the circadian factor Ebony rescued circadian behaviors in Drosophila ${ }^{54}$. These studies suggest that manipulating astrocyte signaling in the SCN can drastically modulate circadian locomotion. It is especially notable that astrocytic circadian control alone is sufficient to drive circadian behavior and that dysfunctional astrocytic circadian control alone is sufficient to break circadian behavior. In both cases, astrocytic outputs completely overshadow the effect of neuronal outputs. These results suggest that astrocytes are not just complementary to neurons but instead are some of the most important players in controlling circadian behaviors.

Beyond circadian locomotive behaviors, several studies have suggested that controlling astrocyte signaling also affects sleeping patterns. Sleep homeostasis is maintained by adenosine accumulation, which is why caffeine, an adenosine receptor antagonist, promotes wakefulness ${ }^{55}$. The source of this adenosine was assumed to be neuronal. However, several studies have clearly demonstrated that 
this adenosine comes from astrocytes. Not surprisingly, one of the most well-known gliotransmitters is ATP. Astrocytic exocytosis via the SNARE complex can be disturbed by introducing a dominant-negative version (dnSNARE), resulting in impaired gliotransmission. When astrocytic dnSNARE was systematically expressed in mice, sleep homeostasis was impaired, and cortical electroencephalogram (EEG) recordings showed attenuated slow wave activity following sleep deprivation ${ }^{56}$. An increase in slow waves is a normal compensatory response to accumulating sleep pressure. The attenuated slow wave power was partially rescued when the A1 receptor antagonist cyclopentyltheophylline was applied intraventricularly, which indicates that purinergic gliotransmission contributes to sleep homeostasis ${ }^{56}$. Alternatively, when cortical astrocytes were optogenetically stimulated in vivo with channelrhodopsin-2 (ChR2), nearby neurons switched to slow wave oscillations ${ }^{57}$. Chemogenetic activation also obtained the same result ${ }^{29}$. It is also notable that astrocytes massively synchronize their $\mathrm{Ca}^{2+}$ activity before neurons drift into slow wave activity ${ }^{58}$. Another study dampened astrocytic $\mathrm{Ca}^{2+}$ activity to induce slow wave oscillations. Expressing the Venus-IPP fusion protein specifically in astrocytes enhanced $\mathrm{IP}_{3}$ to $\mathrm{IP}_{2}$ metabolism and attenuated agonistinduced $\mathrm{Ca}^{2+}$ signaling in astrocytes ${ }^{59}$. When this $\mathrm{IP}_{3} / \mathrm{Ca}^{2+}$ pathway was impaired in hippocampal astrocytes, mice exhibited elevated slow wave power and increased rapideye movement (REM) sleep duration without alterations in their non-REM (NREM) sleep pattern ${ }^{59}$. In addition, optogenetic stimulation of astrocytes in the hypothalamus at $10 \mathrm{~Hz}$ increased both NREM and REM sleep sessions within a 6-h period ${ }^{60}$. In Drosophila, the astrocytic release of a TNF- $\alpha$ homolog is necessary for a sleep deprivationinduced homeostatic response ${ }^{61}$. In that study, purinergic gliotransmission was responsible for the accumulation of sleep pressure. Interestingly, two different astrocytic agents drove seemingly opposing effects. Astrocytic glutamate promoted the physiological circadian rhythm, while astrocytic ATP promoted a wakeful state over a sleep state. However, the astrocytic dnSNARE models used in these studies restricts astrocytic gliotransmission globally. Although the in vitro data suggest that the hippocampus is the region responsible for promoting the wake state, this has not been confirmed in vivo. It is also interesting to note that purinergic gliotransmission is also responsible for negative effects that accompany disruption of the circadian cycle, such as impaired learning and memory, which will be further discussed in a later section.

\section{Astrocytes and cognition}

Cognition, learning, and memory are some of the biggest mysteries in the field of neuroscience. Although significant progress has been made in understanding how we form and recall memories, numerous aspects of memory are yet to be elucidated. Several studies suggest that astrocytes contribute to this process, and acknowledging their roles would advance our understanding of cognitive processes in general. There is accumulating evidence that astrocytes are critically involved in memory formation through various mechanisms, such as by selectively strengthening synapses. Martin-Fernandez et al. used the endocannabinoid and DREADD systems to chemogenetically manipulate astrocytes in the medial subdivision of the central amygdala $(\mathrm{CeM})^{62}$. Astrocytes in this region respond to endogenous cannabinoids via astrocytic cannabinoid receptor type 1 (CB1R) to have elevated $\mathrm{Ca}^{2+}$ levels. This then decreases the excitatory input from the basolateral amygdala (BLA) to the $\mathrm{CeM}^{62}$. Astrocytic expression of stimulatory Gq-DREADD hM3D in this area also causes elevated $\mathrm{Ca}^{2+}$ levels and similar neurophysiological changes. Therefore, both endocannabinoids and Gq DREADD activation of CeM astrocytes caused mice to be completely incapable of forming fear memories in a cued fear-conditioning task ${ }^{62}$. The study also found that elevated $\mathrm{Ca}^{2+}$ levels in astrocytestriggered ATP release and modulated A1R/A2R activities to selectively enhance inhibitory synapses in the $\mathrm{CeM}^{62}$. In several other studies, an astrocytic dnSNARE mouse model was employed to show that gliotransmitters are involved in memory formation. Regulating astrocytic ATP release with dnSNARE alleviated post-sleep deprivation memory impairment in object recognition tests by rescuing synaptic plasticity in the hippocampus ${ }^{56,63}$. This implies that astrocytic ATP not only forces the awake state when one is deprived of sleep but also somewhat eliminates the negative consequences of sleep deprivation. Interestingly, these mice did not seem to accumulate sleep pressure, as if they did not require sleep. In addition to ATP, activity-dependent D-serine is absent in the hippocampus in astrocytic dnSNARE mice ${ }^{64}$. D-serine is an endogenous coagonist of NMDA receptors (NMDARs) at CA3-CA1 synapses. dnSNARE mice performed poorly in contextual fear memory, which was rescued by external D-serine administration ${ }^{64}$. More recent studies have also demonstrated that astrocytic $\mathrm{D}$-serine release depends on astrocytic CB1 receptor activity. One genetic knockout model (GFAP- $C B_{1}$-KO) showed reduced NMDAR activesite occupancy, reduced in vivo hippocampal LTP, and impaired object recognition memory ${ }^{65}$. Along with gene knockout, optogenetic and chemogenetic activation of CA1 astrocytic signaling was also sufficient to enhance memory formation in a context-dependent manner for contextual fear memory and the T-maze ${ }^{66}$. In that study, Opto-Gq and Gq-DREADD hM3D were employed to demonstrate that astrocytic stimulation is sufficient to promote LTP in the hippocampus. Although neuronal stimulation of the area impaired memory performance, 
astrocyte stimulation enhanced it. However, astrocytic memory enhancement was situational. When astrocyte stimulation occurred with learning itself, cognitive augmentation occurred ${ }^{66}$. The effects of NMDAR activity shown in that study imply that D-serine is involved in the de novo potentiation of CA3 to CA1 synapses ${ }^{66}$. By intervening in astrocyte cell signaling through various methods, multiple studies have found a correlation between astrocyte activity and memory formation. This relationship appears to involve gliotransmission, notably transmission involving ATP and D-serine, in the hippocampus. Multiple studies agree that astrocytic D-serine release occurs in the hippocampus as the Gq pathway is activated. This D-serine critically modulates LTP formation in this area. However, gliotransmission is dysfunctional in some pathophysiological models. In a mouse model of Alzheimer's disease (AD), astrocytes release excessive GABA through the Best1 channel. The cognitive symptoms exhibited by a mouse model of AD were alleviated by oral administration of selegilline, which blocks monoamine oxidase B activity, ultimately blocking astrocytic GABA synthesis ${ }^{67}$. Therefore, this study identified GABA as another potential gliomodulator of cognition. In vitro data suggest that hippocampal activities can be rescued by either inhibiting GABA synthesis or blocking the Best1 channel. However, a GABA synthesis inhibitor was not administered in vivo in the above-mentioned study. Therefore, further confirmation of this phenomenon is necessary.

The studies that we have described thus far have concluded that astrocyte signaling results in gliotransmitter release that affects synaptic function. Another interesting hypothesis is that rather than modulating synapses, astrocytes modulate the activity of nearby neurons by supplying them with energy in the form of lactate. The significance of lactate transportation in the CNS was first described in $2011^{68}$. Since then, multiple groups have investigated the in vivo behavioral role of the lactate transport system in astrocytes. Glycolysis in astrocytes results in lactate release via monocarboxylate transporters (MCTs) 1 and 4 (astrocytes) to MCT2 (neurons). One study found that disruption of the astrocytic lactate transporters MCT1 and MCT4 in the hippocampus caused amnesia ${ }^{68}$. Adrenergic signaling of the area triggers astrocytic release of lactate, and $\beta_{2}$-adrenergic receptors $\left(\beta_{2} \mathrm{AR}\right)$ are expressed by hippocampal astrocytes. When exposed to a $\beta_{2} \mathrm{AR}$ antagonist, mice failed to exhibit long-term inhibitory avoidance memory formation $\operatorname{tasks}^{69}$. This effect was rescued when lactate was supplied locally, indicating that these receptors are responsible for learning-evoked lactate release from astrocytes in the region ${ }^{69}$. In accordance with this result, another study administered a $\beta_{2} \mathrm{AR}$ agonist by injection over multiple days. This intervention activated downstream signaling pathways, including pathways associated with lactate metabolism and transportation, and greatly improved the performance of the animals in the Morris water maze test $^{70}$. In the hippocampus, astrocyte-neuron lactate transfer is important for the consolidation/reconsolidation of appetitive conditioning. Infusion of glycogen phosphorylase into the BLA of rats impaired astrocyteneuron lactate transportation. This effect not only prevented rats from forming the new cocaine-based conditioned place preferences but also disturbed existing preferences $^{71}$. These studies collectively suggest that, in response to external stimuli, astrocytes undergo glycolysis to produce lactate and release it to adjacent neurons. Astrocytic lactate plays a crucial role in forming and recalling memories across multiple brain regions, including the hippocampus and amygdala. Combined with previous descriptions, it appears that hippocampal astrocytes modulate learning on multiple levels. Inputs coming into the hippocampus are sensed by local astrocytes. These inputs activate the Gq pathway and lead to $\mathrm{Ca}^{2+}$ elevation. Astrocytes then oversee lactate transportation and local adrenergic and D-serine signaling, which promote neuronal activity and synaptic plasticity in the area. An increasing amount of in vivo evidence suggests that hippocampal astrocytes are important for learning and memory. This evidence underscores the importance of fully elucidating neuron-astrocyte communication to solve one of the biggest mysteries in neuroscience.

\section{Astrocytes and mood-associated behaviors}

Soon after astrocytes were found to react to nearby neuronal activity, they were also seen to respond rapidly to pathophysiological states in the brain. Traditionally, astrocytes were thought to mainly respond to physical damage in the brain, such as ischemia or neurodegenerative diseases (such as AD). Recent advancements in the understanding of astroglial functions suggest that astrocytes are also involved in neuropsychiatric pathophysiology. For instance, astrocytes display altered morphology when neuropathic pain is induced ${ }^{43}$. Similarly, major depressive disorder is associated with a reduced number and density of astrocytes, and astrocytes exhibit cell hypotrophy in both rodents and humans ${ }^{72}$. Cao et al. ${ }^{73}$ showed that astrocytic signaling can have both depressive and anti-depressive effects on mice. In this study, Itpr $2^{-/-}$ transgenic mice were used to disrupt the $\mathrm{IP}_{3}$ signaling pathways in astrocytes. Astrocytic $\mathrm{Ca}^{2+}$ elevation and ATP release failed to be induced in knockout mice, but neuronal ATP release was unaffected ${ }^{73}$. These mice were susceptible to chronic social defeats and rapidly developed depression-like behaviors. Notably, systemic administration of ATP induced antidepressant-like effects in the mice $^{73}$. In addition, the mice that were previously affected by chronic social defeat stress quickly recovered when 
astrocytic Gq signaling was enhanced by overexpression of the mas-related gene A1, which elevates intracellular $\mathrm{Ca}^{2+}$. These mice show decreased immobility in a forced swim test and increased interaction during social defeat sessions $^{73}$. However, there is contradictory evidence regarding whether $\mathrm{IP}_{3} \mathrm{R} 2$-dependent $\mathrm{Ca}^{2+}$ signaling and gliotransmission induce anxiety or depressive-like behavior $^{74}$. In the above-mentioned study, $\mathrm{IP}_{3} \mathrm{R} 2 \mathrm{KO}$ model mice did not show any abnormalities in the open field test or the tail suspension test. Therefore, given the multiple contradicting results, it remains difficult to clarify the relationship between astrocytic activity and depression. This controversy may be explained by the complex nature of depression and the lack of decisive behavior tests that are available to evaluate it.

In contrast, another mood-associated behavioral disorder is well known to be governed by astrocytes. Obsessive-compulsive disorder (OCD) is an anxiety disorder that is characterized by repetitive behaviors. The most prominent theory regarding the underlying mechanism of OCD is a dysregulated excitation to inhibition (E/I) ratio. As astrocytes are homeostatic controllers, several studies have found that astrocytes are involved in repetitive behavior. Astrocytes express glutamate transporter 1 (GLT1) to regulate the E/I balance. One study found that astrocyte-specific conditional GLT1 knockout mice (GLAST ${ }^{\mathrm{CreERT} 2 /+} / \mathrm{GLT}^{\mathrm{flx} / \mathrm{flx}}$ ) exhibited pathological levels of repetitive behaviors to the point of injuring themselves ${ }^{75}$. Systematic administration of NMDA antagonist alleviated this symptom, which suggests that astrocytic glutamate transportation via GLT1 plays a critical role in controlling repetitive behaviors ${ }^{75}$. In another study, a modified isoform of the human plasma membrane calcium pump (hPMCA2w/b) was exogenously expressed specifically in striatal astrocytes ${ }^{45}$. This calcium pump constitutively extrudes cytosolic $\mathrm{Ca}^{2+}$, which renders cells almost devoid of calcium. Although hPMCA2w/b mice did not display any anxiety or depression-like phenotypes, they demonstrated extensive self-grooming behavior when they were left alone ${ }^{45}$. As a putative underlying mechanism of such behavioral alterations, in vivo $\mathrm{Ca}^{2+}$ imaging revealed that medium spiny neuron (MSN) activity was significantly dampened when nearby astrocyte $\mathrm{Ca}^{2+}$ signaling was abolished. Blocking the astrocytic GABA transporter GAT-3 also partially rescued the phenotype. This result suggests that astrocytic $\mathrm{Ca}^{2+}$ signaling is responsible for tonic GABA inhibition in the striatum ${ }^{40}$. Interestingly, GABA release from MSNs results in acute behavioral hyperactivity and disrupted attention, which is rescued by inhibition of the astrocytic $\mathrm{GABA}_{\mathrm{B}}-\mathrm{Gi}$ pathway ${ }^{76}$. Artificially activating this pathway also upregulates thrombospondin-1 in astrocytes, which in turn increases excitatory synapses and enhances cortical synaptic transmission ${ }^{76}$. These results suggest that MSNs and astrocytes in the striatum reciprocally exchange GABA signals. Additionally, tonic GABA signals that result from elevated astrocytic $\mathrm{Ca}^{2+}$ inhibit the activity of MSNs to produce repetitive behaviors (Table 1 ).

\section{Other behaviors}

Direct behavior control by astrocytes is not limited to the circadian rhythm, memory, and mood. Multiple studies have controlled various aspects of astrocyte signaling to produce diverse observable behaviors. Just as astrocytes control homeostasis of the circadian rhythm, they also regulate nutritional homeostasis. DREADDmediated modulation of astrocytes in the arcuate nucleus using Gq-DREADD hM3D altered appetite in mice ${ }^{77,78}$. The mice demonstrated an acute increase in food consumption following $\mathrm{CNO}$ administration. Activated astrocytes also specifically interact with adjacent $\mathrm{AgRP} / \mathrm{NPY}$ neurons via adenosine $\mathrm{A}_{1}$ receptors to modulate food intake ${ }^{78}$. Yang et al. ${ }^{77}$ found the opposite effect to be true when they "inhibited" astrocyte activity using Gi-DREADD hM4D. However, multiple other reports have found that the Gi pathway also results in elevated $\mathrm{Ca}^{2+}$ levels in astrocytes ${ }^{15,28,29}$. The Gq and $\mathrm{Gi}$ pathways result in different degrees of $\mathrm{Ca}^{2+}$ elevation ${ }^{15}$. However, it remains to be determined whether the $\mathrm{Gi}$ pathway truly is "inhibitory" and reverses the effects of the Gq pathway. In addition to feeding behavior, astrocytes are also involved in reward and aversive behavior. We have previously discussed a study showing how endocannabinoid input to astrocytes is necessary for reward-based learning ${ }^{62}$. Optogenetically stimulating astrocytes in the ventral tegmental area (VTA) with ChR2 resulted in clear, real-time avoidance of the "lightON" area during a real-time place preference test ${ }^{79}$. Such avoidance behavior was absent in GLT1-cKO VTA Astrocyte mice, indicating that astrocytic glutamate transport in the area is crucial for modulating aversive behavior ${ }^{79}$. In that study, astrocytes were shown to adjust the glutamatergic input to local GABA neurons that inhibit dopamine neurons in turn ${ }^{79}$. Although the brain subregions examined in these studies differed (the amygdala vs. the VTA), they both demonstrate that astrocytic signaling is crucial for the development of healthy approach-avoidance behavior. Another study suggested that proper motor skill learning depends on astrocyte signaling. When astrocyte $\mathrm{Ca}^{2+}$ signaling was attenuated via $\mathrm{IP}_{3} \mathrm{R} 2$ knockout or the astrocyte-specific metabolic inhibitor fluorocitrate, mice demonstrated poor performance in motor skill learning ${ }^{80}$. In that study, both $\mathrm{IP}_{3} \mathrm{R} 2$ deficiency and fluorocitrate treatment impaired LTP-like activity in the motor cortex. This impairment prevented motor skill training-induced glutamate transmission ${ }^{80}$. These findings indicate that astrocytic 


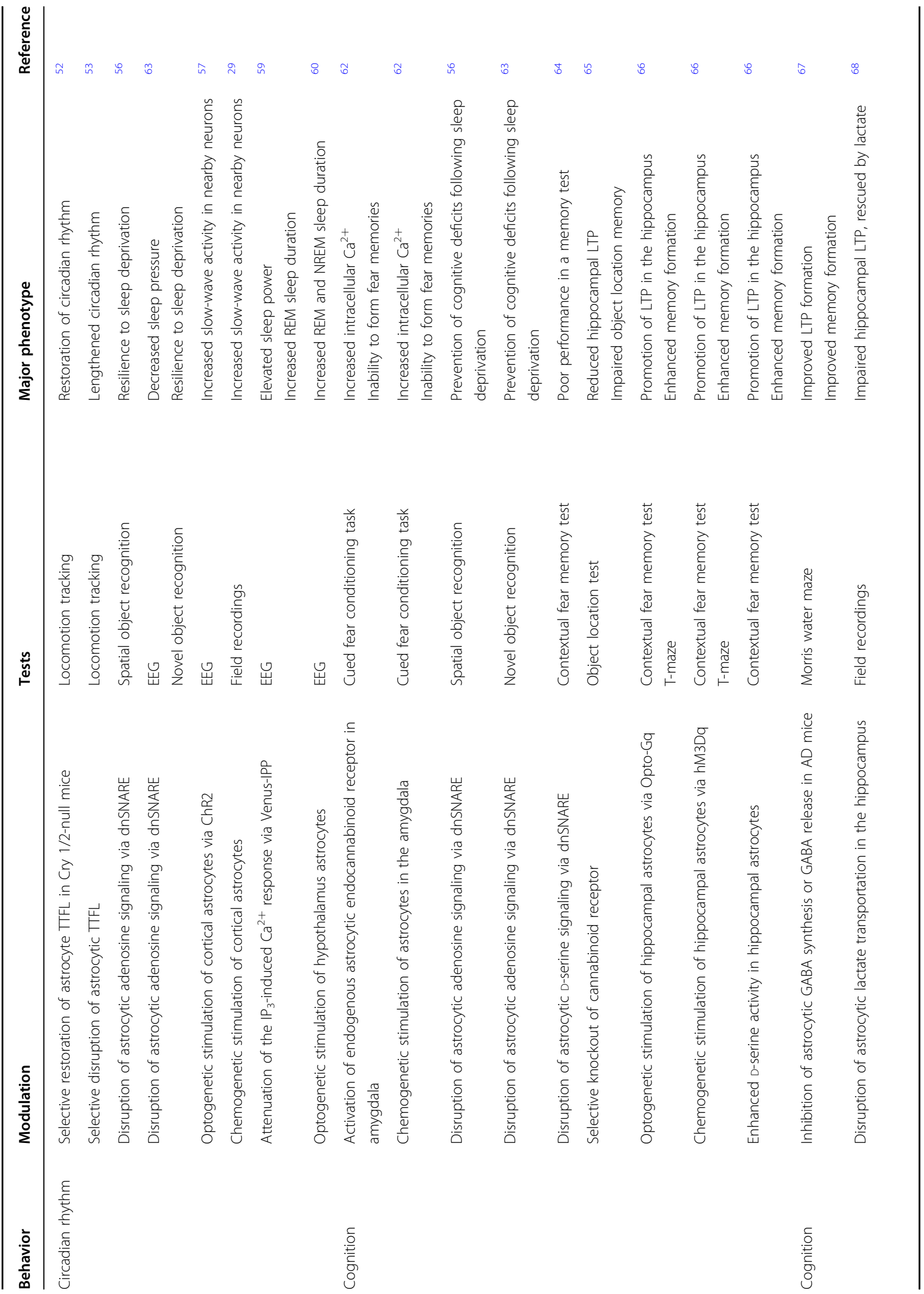




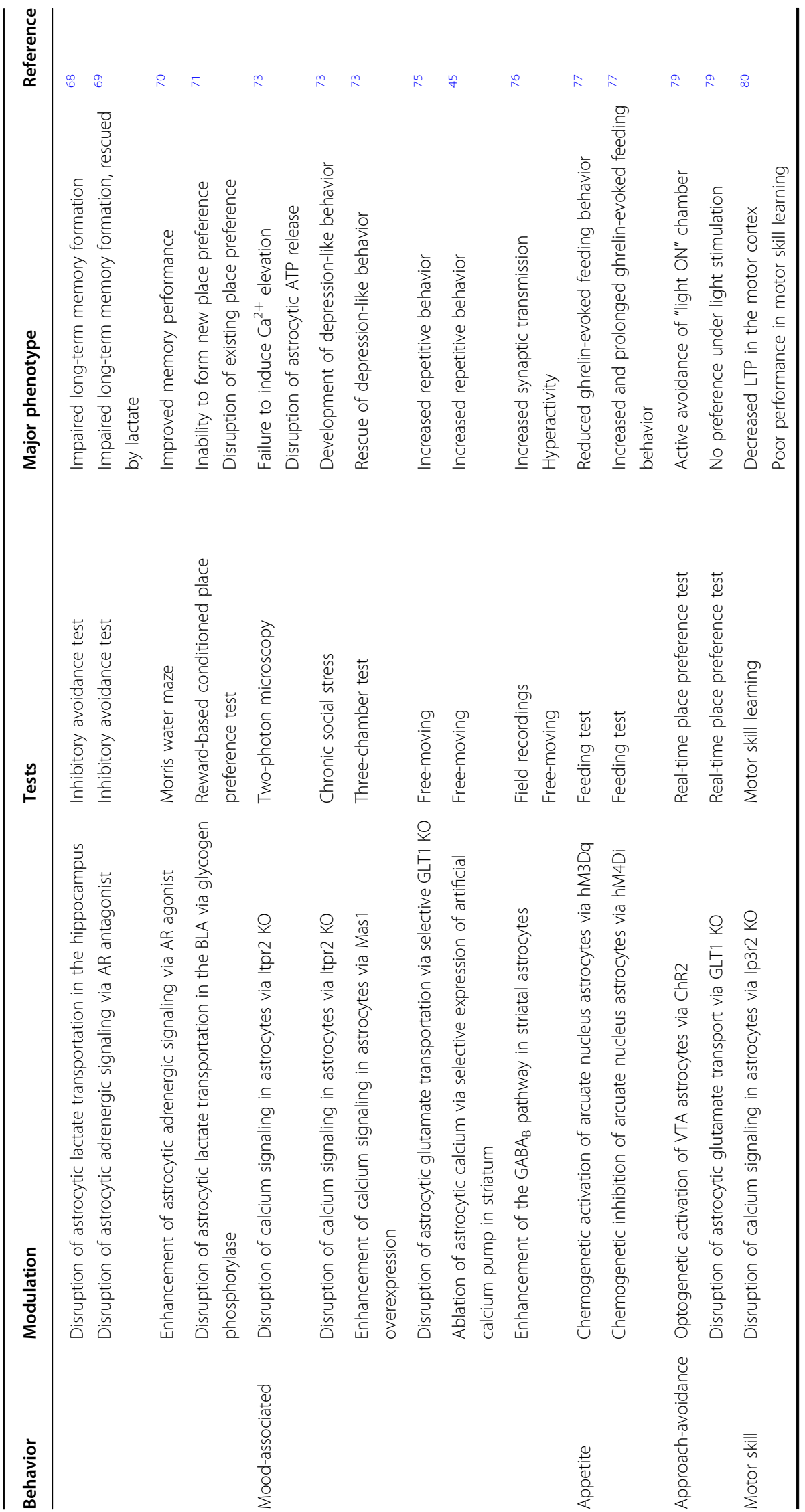


$\mathrm{Ca}^{2+}$ is implicated not only in decisive memory formation but also in procedural memory formation.

\section{Conclusion}

The diverse behavioral roles of astrocytes in the CNS are becoming more apparent. In the last decade, astrocytes have emerged as critical modulators of information processing in the brain. Although numerous reports have been shifting attention from neurons to astrocytes, the precise in vivo functions of astrocytes have only begun to be decoded. This review discusses recent efforts aimed at elucidating how the astrocyte signaling process is carried out and presents wide-ranging evidence that controlling astrocyte activity can directly affect animal behaviors. Endeavors to decipher the star codings are relatively new, and our current understanding of astrocyte physiology is limited. Although assessing astrocyte function in behaviors in vivo is challenging, new technologies and more accurate models are constantly arising to help researchers precisely monitor and manipulate astrocyte activities in vivo, which will aid in establishing and validating more refined theories of behavioral neuroscience based on neuron-glia communication. The future of research on astrocytes in behavioral neuroscience promises to be fascinating and will likely accompany meaningful real-life applications in fields such as clinical medicine.

\section{Acknowledgements}

This work was supported by the Samsung Science \& Technology Foundation (SSTF-BA150208282).

\section{Conflict of interest}

The authors declare that they have no conflict of interest.

\section{Publisher's note}

Springer Nature remains neutral with regard to jurisdictional claims in published maps and institutional affiliations.

Received: 24 December 2019 Revised: 30 April 2020 Accepted: 8 June 2020. Published online: 15 July 2020

\section{References}

1. von Bartheld, C. S., Bahney, J. \& Herculano-Houzel, S. The search for true numbers of neurons and glial cells in the human brain: a review of 150 years of cell counting. J. Comp. Neurol. 524, 3865-3895 (2016).

2. Popovich, P. G., Jakeman, L. B. \& McTigue, D. M. Glial responses to injury Encycl. Neurosci. 853-859, https://doi.org/10.1016/B978-008045046-9.00018-8 (2009).

3. Tsacopoulos, M. \& Magistretti, P. J. Metabolic coupling between glia and neurons. J. Neurosci. 16, 877-885 (1996)

4. Cornell-Bell, A. H., Finkbeiner, S. M., Cooper, M. S. \& Smith, S. J. Glutamate induces calcium waves in cultured astrocytes: long-range glial signaling. Science 247, 470-473 (1990).

5. Smith, S. J. Do astrocytes process neural information? Prog. Brain Res. 94 119-136 (1992)

6. Smith, S. J. Neural signalling. Neuromodulatory astrocytes. Curr. Biol. 4 807-810 (1994)

7. Goldman, N. et al. Adenosine A1 receptors mediate local anti-nociceptive effects of acupuncture. Nat. Neurosci. 13, 883-888 (2010).
8. Milligan, E. D. \& Watkins, L. R. Pathological and protective roles of glia in chronic pain. Nat. Rev. Neurosci. 10, 23-36 (2009).

9. Voskuhl, R. R. et al. Reactive astrocytes form scar-like perivascular barriers to leukocytes during adaptive immune inflammation of the CNS. J. Neurosci. $\mathbf{2 9}$ 11511-11522 (2009)

10. Becerra-Calixto, A. \& Cardona-Gomez, G. P. The role of astrocytes in neuroprotection after brain stroke: potential in cell therapy. Front. Mol. Neurosci. 10, 88 (2017).

11. Heuser, K. et al. Ca2+ signals in astrocytes facilitate spread of epileptiform activity. Cereb. Cortex 28, 4036-4048 (2018).

12. De Carlos, J. A. \& Borrell, J. A historical reflection of the contributions of Cajal and Golgi to the foundations of neuroscience. Brain Res. Rev. 55, 8-16 (2007).

13. Halassa, M. M., Fellin, T., Takano, H., Dong, J. H. \& Haydon, P. G. Synaptic islands defined by the territory of a single astrocyte. J. Neurosci. 27, 6473-6477 (2007).

14. Tanaka, M. et al. Astrocytic Ca2+ signals are required for the functional integrity of tripartite synapses. Mol. Brain 6, 6 (2013).

15. Chai, H. et al. Neural circuit-specialized astrocytes: transcriptomic, proteomic, morphological, and functional evidence. Neuron 95, 531-549 e539 (2017).

16. Oliveira, J. F., Sardinha, V. M., Guerra-Gomes, S., Araque, A. \& Sousa, N. Do stars govern our actions? Astrocyte involvement in rodent behavior. Trends Neurosci. 38, 535-549 (2015).

17. Farhy-Tselnicker, I. \& Allen, N. J. Astrocytes, neurons, synapses: a tripartite view on cortical circuit development. Neural Dev. 13, 7 (2018).

18. Dani, J. W., Chernjavsky, A. \& Smith, S. J. Neuronal activity triggers calcium waves in hippocampal astrocyte networks. Neuron 8, 429-440 (1992).

19. Morais, T. P. Coelho, D. Vaz, S. H. Sebastiao, A. M. \& Valente, C. A. Glycine receptor activation impairs ATP-induced calcium transients in cultured cortical astrocytes. Front. Mol. Neurosci. 10, 444 (2017).

20. Fujii, Y., Maekawa, S. \& Morita, M. Astrocyte calcium waves propagate proximally by gap junction and distally by extracellular diffusion of ATP released from volume-regulated anion channels. Sci. Rep. 7, 13115 (2017).

21. Tong, X., Shigetomi, E., Looger, L. L. \& Khakh, B. S. Genetically encoded calcium indicators and astrocyte calcium microdomains. Neuroscientist 19, 274-291 (2013).

22. Smith, N. A. et al. Fluorescent $\mathrm{Ca}(2+)$ indicators directly inhibit the Na,K-ATPase and disrupt cellular functions. Sci. Signal. 11, https://doi.org/10.1126/scisignal. aal2039 (2018).

23. Bootman, M. D., Allman, S., Rietdorf, K. \& Bultynck, G. Deleterious effects of calcium indicators within cells; an inconvenient truth. Cell Calcium 73, 82-87 (2018).

24. Yang, Y. et al. Improved calcium sensor GCaMP-X overcomes the calcium channel perturbations induced by the calmodulin in GCaMP. Nat. Commun. 9, 1504 (2018)

25. Inoue, M. et al. Rational engineering of XCaMPs, a multicolor GECl suite for in vivo imaging of complex brain circuit dynamics. Cell 177, 1346-1360 e1324 (2019).

26. Shigetomi, E., Patel, S. \& Khakh, B. S. Probing the complexities of astrocyte calcium signaling. Trends Cell Biol. 26, 300-312 (2016).

27. Ding, F. et al. alpha1-Adrenergic receptors mediate coordinated Ca2+ signaling of cortical astrocytes in awake, behaving mice. Cell Calcium 54, 387-394 (2013).

28. Mariotti, L., Losi, G., Sessolo, M., Marcon, I. \& Carmignoto, G. The inhibitory neurotransmitter GABA evokes long-lasting $\mathrm{Ca}(2+)$ oscillations in cortical astrocytes. Glia 64, 363-373 (2016).

29. Durkee, C. A. et al. Gi/o protein-coupled receptors inhibit neurons but activate astrocytes and stimulate gliotransmission. Glia 67, 1076-1093 (2019).

30. Petravicz, J., Fiacco, T. A. \& McCarthy, K. D. Loss of IP3 receptor-dependent Ca2 + increases in hippocampal astrocytes does not affect baseline CA1 pyramidal neuron synaptic activity. J. Neurosci. 28, 4967-4973 (2008).

31. Takata, N. et al. Cerebral blood flow modulation by Basal forebrain or whisker stimulation can occur independently of large cytosolic $\mathrm{Ca} 2+$ signaling in astrocytes. PLOS ONE 8, e66525 (2013).

32. Srinivasan, $\mathrm{R}$. et al. $\mathrm{Ca}(2+)$ signaling in astrocytes from $\operatorname{lp} 3 \mathrm{r} 2(-/-)$ mice in brain slices and during startle responses in vivo. Nat. Neurosci. 18, 708-717 (2015).

33. Shigetomi, E., Tong, X., Kwan, K. Y., Corey, D. P. \& Khakh, B. S. TRPA1 channels regulate astrocyte resting calcium and inhibitory synapse efficacy through GAT-3. Nat. Neurosci. 15, 70-80 (2011).

34. Ho, K. W., Lambert, W. S. \& Calkins, D. J. Activation of the TRPV1 cation channe contributes to stress-induced astrocyte migration. Glia 62, 1435-1451 (2014). 
35. Agarwal, A. et al. Transient opening of the mitochondrial permeability transition pore induces microdomain calcium transients in astrocyte processes. Neuron 93, 587-605. e587 (2017).

36. Guerra-Gomes, S., Sousa, N., Pinto, L. \& Oliveira, J. F. Functional roles of astrocyte calcium elevations: from synapses to behavior. Front. Cell. Neurosci. 11, 427 (2017).

37. Souza, D. G., Almeida, R. F., Souza, D. O. \& Zimmer, E. R. The astrocyte biochemistry. Semin. Cell Dev. Biol. 95, 142-150 (2019).

38. Ormel, L., Stensrud, M. J., Bergersen, L. H. \& Gundersen, V. VGLUT1 is localized in astrocytic processes in several brain regions. Glia 60, 229-238 (2012).

39. Verkhratsky, A., Matteoli, M., Parpura, V., Mothet, J. P. \& Zorec, R. Astrocytes as secretory cells of the central nervous system: idiosyncrasies of vesicular secretion. EMBO J. 35, 239-257 (2016).

40. Todd, K. J., Darabid, H. \& Robitaille, R. Perisynaptic glia discriminate patterns of motor nerve activity and influence plasticity at the neuromuscular junction. J. Neurosci. 30, 11870-11882 (2010).

41. Shigetomi, E., Jackson-Weaver, O., Huckstepp, R. T., O'Dell, T. J. \& Khakh, B. S. TRPA1 channels are regulators of astrocyte basal calcium levels and long-term potentiation via constitutive D-serine release. J. Neurosci. 33, 10143-10153 (2013).

42. Min, R. \& Nevian, T. Astrocyte signaling controls spike timing-dependent depression at neocortical synapses. Nat. Neurosci. 15, 746-753 (2012).

43. Shigetomi, E., Saito, K., Sano, F. \& Koizumi, S. Aberrant calcium signals in reactive astrocytes: a key process in neurological disorders. Int. J. Mol. Sci. 20 https://doi.org/10.3390/ijms20040996 (2019).

44. Savtchouk, I. \& Volterra, A. Gliotransmission: beyond Black-and-White. J. Neurosci. 38, 14-25 (2018).

45. $\mathrm{Yu}, \mathrm{X}$. et al. Reducing astrocyte calcium signaling in vivo alters striatal microcircuits and causes repetitive behavior. Neuron 99, 1170-1187 e1179 (2018).

46. Figueiredo, M. et al. Optogenetic experimentation on astrocytes. Exp. Physiol. 96, 40-50 (2011).

47. Mederos, S. et al. Melanopsin for precise optogenetic activation of astrocyteneuron networks. Glia 67, 915-934 (2019).

48. Cho, W. H., Barcelon, E. \& Lee, S. J. Optogenetic glia manipulation: possibilities and future prospects. Exp. Neurobiol. 25, 197-204 (2016).

49. Nam, Y. et al. Reversible induction of pain hypersensitivity following optogenetic stimulation of spinal astrocytes. Cell Rep. 17, 3049-3061 (2016).

50. Doherty, C. J. \& Kay, S. A. Circadian control of global gene expression patterns. Annu. Rev. Genet. 44, 419-444 (2010).

51. Barca Mayo, O., Berdondini, L. \& De Pietri Tonelli, D. Astrocytes and circadian rhythms: an emerging astrocyte-neuron synergy in the timekeeping system. Methods Mol. Biol. 1938, 131-154 (2019).

52. Brancaccio, M. et al. Cell-autonomous clock of astrocytes drives circadian behavior in mammals. Science 363, 187-192 (2019).

53. Tso, C. F. et al. Astrocytes regulate daily rhythms in the suprachiasmatic nucleus and behavior. Curr. Biol. 27, 1055-1061 (2017).

54. Suh, J. \& Jackson, F. R. Drosophila ebony activity is required in glia for the circadian regulation of locomotor activity. Neuron 55, 435-447 (2007).

55. Brown, R. E., Basheer, R., McKenna, J. T., Strecker, R. E. \& McCarley, R. W. Control of sleep and wakefulness. Physiol. Rev. 92, 1087-1187 (2012).

56. Halassa, M. M. et al. Astrocytic modulation of sleep homeostasis and cognitive consequences of sleep loss. Neuron 61, 213-219 (2009).

57. Poskanzer, K. E. \& Yuste, R. Astrocytes regulate cortical state switching in vivo. Proc. Natl Acad. Sci. USA 113, E2675-E2684 (2016).

58. Szabo, Z. et al. Extensive astrocyte synchronization advances neuronal coupling in slow wave activity in vivo. Sci. Rep. 7, 6018 (2017).
59. Foley, J. et al. Astrocytic IP3/Ca(2+) signaling modulates theta rhythm and REM sleep. Front. Neural Circuits 11, 3 (2017).

60. Pelluru, D., Konadhode, R. R., Bhat, N. R. \& Shiromani, P. J. Optogenetic stimulation of astrocytes in the posterior hypothalamus increases sleep at night in C57BL/6J mice. Eur. J. Neurosci. 43, 1298-1306 (2016).

61. Vanderheyden, W. M. et al. Astrocyte expression of the Drosophila TNF-alpha homologue, Eiger, regulates sleep in flies. PLoS Genet. 14, e1007724 (2018).

62. Martin-Fernandez, M. et al. Synapse-specific astrocyte gating of amygdalarelated behavior. Nat. Neurosci. 20, 1540-1548 (2017).

63. Florian, C., Vecsey, C. G., Halassa, M. M., Haydon, P. G. \& Abel, T. Astrocytederived adenosine and $A 1$ receptor activity contribute to sleep loss-induced deficits in hippocampal synaptic plasticity and memory in mice. J. Neurosci. 31, 6956-6962 (2011)

64. Papouin, T., Dunphy, J. M., Tolman, M., Dineley, K. T. \& Haydon, P. G. Septal cholinergic neuromodulation tunes the astrocyte-dependent gating of hippocampal NMDA receptors to wakefulness. Neuron 94, 840-854 e847 (2017).

65. Robin, L. M. et al. Astroglial CB1 receptors determine synaptic D-serine availability to enable recognition memory. Neuron 98, 935-944 e935 (2018).

66. Adamsky, A. et al. Astrocytic activation generates de novo neuronal potentiation and memory enhancement. Cell 174, 59-71 e14 (2018).

67. Jo, S. et al. GABA from reactive astrocytes impairs memory in mouse models of Alzheimer's disease. Nat. Med. 20, 886-896 (2014).

68. Suzuki, A. et al. Astrocyte-neuron lactate transport is required for long-term memory formation. Cell 144, 810-823 (2011).

69. Gao, V. et al. Astrocytic beta2-adrenergic receptors mediate hippocampal long-term memory consolidation. Proc. Natl Acad. Sci. USA 113, 8526-8531 (2016).

70. Dong, J. H. et al. Adaptive activation of a stress response pathway improves learning and memory through Gs and beta-arrestin-1-regulated lactate metabolism. Biol. Psychiatry 81, 654-670 (2017).

71. Boury-Jamot, B. et al. Disrupting astrocyte-neuron lactate transfer persistently reduces conditioned responses to cocaine. Mol. Psychiatry 21, 1070-1076 (2016).

72. Wang, Q., Jie, W., Liu, J. H., Yang, J. M. \& Gao, T. M. An astroglial basis of major depressive disorder? An overview. Glia 65, 1227-1250 (2017).

73. Cao, X. et al. Astrocyte-derived ATP modulates depressive-like behaviors. Nat. Med. 19, 773-777 (2013)

74. Petravicz, J., Boyt, K. M. \& McCarthy, K. D. Astrocyte IP3R2-dependent Ca(2+) signaling is not a major modulator of neuronal pathways governing behavior Front. Behav. Neurosci. 8, 384 (2014).

75. Aida, T. et al. Astroglial glutamate transporter deficiency increases synaptic excitability and leads to pathological repetitive behaviors in mice. Neuropsychopharmacology 40, 1569-1579 (2015).

76. Nagai, J. et al. Hyperactivity with disrupted attention by activation of an astrocyte synaptogenic cue. Cell 177, 1280-1292 e1220 (2019).

77. Yang, L., Qi, Y. \& Yang, Y. Astrocytes control food intake by inhibiting AGRP neuron activity via adenosine A1 receptors. Cell Rep. 11, 798-807 (2015).

78. Chen, N. et al. Direct modulation of GFAP-expressing glia in the arcuate nucleus bi-directionally regulates feeding. Elife 5, https://doi.org/10.7554/ elife.18716 (2016).

79. Gomez, J. A. et al. Ventral tegmental area astrocytes orchestrate avoidance and approach behavior. Nat. Commun. 10, 1455 (2019).

80. Padmashri, R., Suresh, A., Boska, M. D. \& Dunaevsky, A. Motor-skill learning is dependent on astrocytic activity. Neural Plast. 2015, 938023 (2015). 